\title{
Waon Therapy Upregulates Hsp90 and Leads to Angiogenesis Through the Akt-Endothelial Nitric Oxide Synthase Pathway in Mouse Hindlimb Ischemia
}

\author{
Takahiro Miyauchi, MD; Masaaki Miyata, MD; Yoshiyuki Ikeda, MD; Yuichi Akasaki, MD; \\ Narisato Hamada, MD; Takahiro Shirasawa, MD; Yuko Furusho, MD; Chuwa Tei, MD
}

\begin{abstract}
Background: Thermal therapy, namely Waon therapy, has previously been reported to regulate nitric oxide (NO) and endothelial NO synthase (eNOS) and augment ischemia-induced angiogenesis in mice and improve limb ischemia in patients with peripheral artery disease. The aim of this study was to clarify the precise mechanism by which Waon therapy augments angiogenesis in mice with hindlimb ischemia.

Methods and Results: Unilateral hindlimb ischemia was induced in apolipoprotein E-deficient mice and Waon therapy was performed for 5 weeks. Heat shock protein 90 (Hsp90), phosphorylated-Akt, and phosphorylated-eNOS were detected in arterial endothelial cells of ischemic hindlimbs and all were upregulated by Waon therapy compared to controls. Waon therapy also increased serum concentrations of nitrite and nitrate. Capillary density and the ischemic limb/normal side blood perfusion ratio monitored by laser Doppler perfusion imaging in the Waon therapy group were significantly increased beyond those in the control group. The effect of Waon therapy on angiogenesis through the activation of the Hsp90/Akt/eNOS pathway was attenuated by the administration of a Hsp90 inhibitor.
\end{abstract}

Conclusions: It is suggested that Waon therapy upregulates Hsp90, which contributes to the activation of the Akt/eNOS/NO pathway, and induces angiogenesis in mice with hindlimb ischemia. (Circ $J$ 2012; 76: 1712-1721)

Key Words: Angiogenesis; eNOS; Hsp90; Waon therapy

W e have developed a form of thermal therapy, namely Waon therapy, which differs from the traditional sauna. ${ }^{1}$ Waon therapy is performed as follows: patients are placed in a dry sauna for $15 \mathrm{~min}$ at $60^{\circ} \mathrm{C}$, and then subsequently kept in a supine position on a bed with sufficient warmth provided by blankets for an additional $30 \mathrm{~min}$ outside the sauna room. ${ }^{2}$ We have previously reported that Waon therapy improves hemodynamics, cardiac function, ventricular arrhythmias, vascular endothelial function, neuro-hormonal factors, sympathetic nervous system, oxidative stress, clinical symptoms, and prognosis in patients with chronic heart failure (CHF). ${ }^{1-10}$

\section{Editorial $\mathrm{p} 1593$}

In addition, we have previously reported that Waon therapy improves symptoms, status, and blood flow in patients with peripheral arterial disease (PAD). ${ }^{11-13}$ Furthermore, we reported that repeated Waon therapy increases endothelial nitric oxide (NO) synthase (eNOS) protein expression, blood flow, and capillary density in a mouse model of hind limb ischemia, and concluded that the increased NO plays a key role in angiogenesis induced by Waon therapy. ${ }^{14}$

Heat shock protein 90 (Hsp90) is an abundant and ubiquitous molecular chaperone that facilitates the folding of a variety of proteins and maturation of other proteins. ${ }^{15} \mathrm{Hsp} 90$ forms a complex with Akt and is responsible for the phosphorylation of Akt. ${ }^{16}$ Phosphorylated Akt directly phosphorylates eNOS, which leads to the production of NO. ${ }^{17}$

NO, constitutively produced by eNOS, plays important roles in vascular biology including regulation of vascular tone and blood pressure, as well as the regulation of angiogenesis. ${ }^{18,19}$ PAD patients are characterized by reduced systemic NO bioactivity. The impaired neovascularization in mice lacking eNOS is related to a defect in progenitor cell mobilization. eNOS expressed by bone marrow stromal cells influences recruitment of stem and progenitor cells. ${ }^{20}$

Although we have reported that Waon therapy is beneficial for PAD, the mechanism through which Waon therapy activates eNOS has not been identified. We hypothesized that

Received August 17, 2011; revised manuscript received February 23, 2012; accepted February 26, 2012; released online April 6, 2012 Time for primary review: 19 days

Department of Cardiovascular, Respiratory and Metabolic Medicine, Graduate School of Medicine, Kagoshima University, Kagoshima, Japan

Mailing address: Masaaki Miyata, MD, FAHA, FACC, Department of Cardiovascular, Respiratory and Metabolic Medicine, Graduate School of Medicine, Kagoshima University, 8-35-1 Sakuragaoka, Kagoshima 890-8520, Japan. E-mail: miyatam@m3.kufm.kagoshima-u.ac.jp

ISSN-1346-9843 doi:10.1253/circj.CJ-11-0915

All rights are reserved to the Japanese Circulation Society. For permissions, please e-mail: cj@j-circ.or.jp 


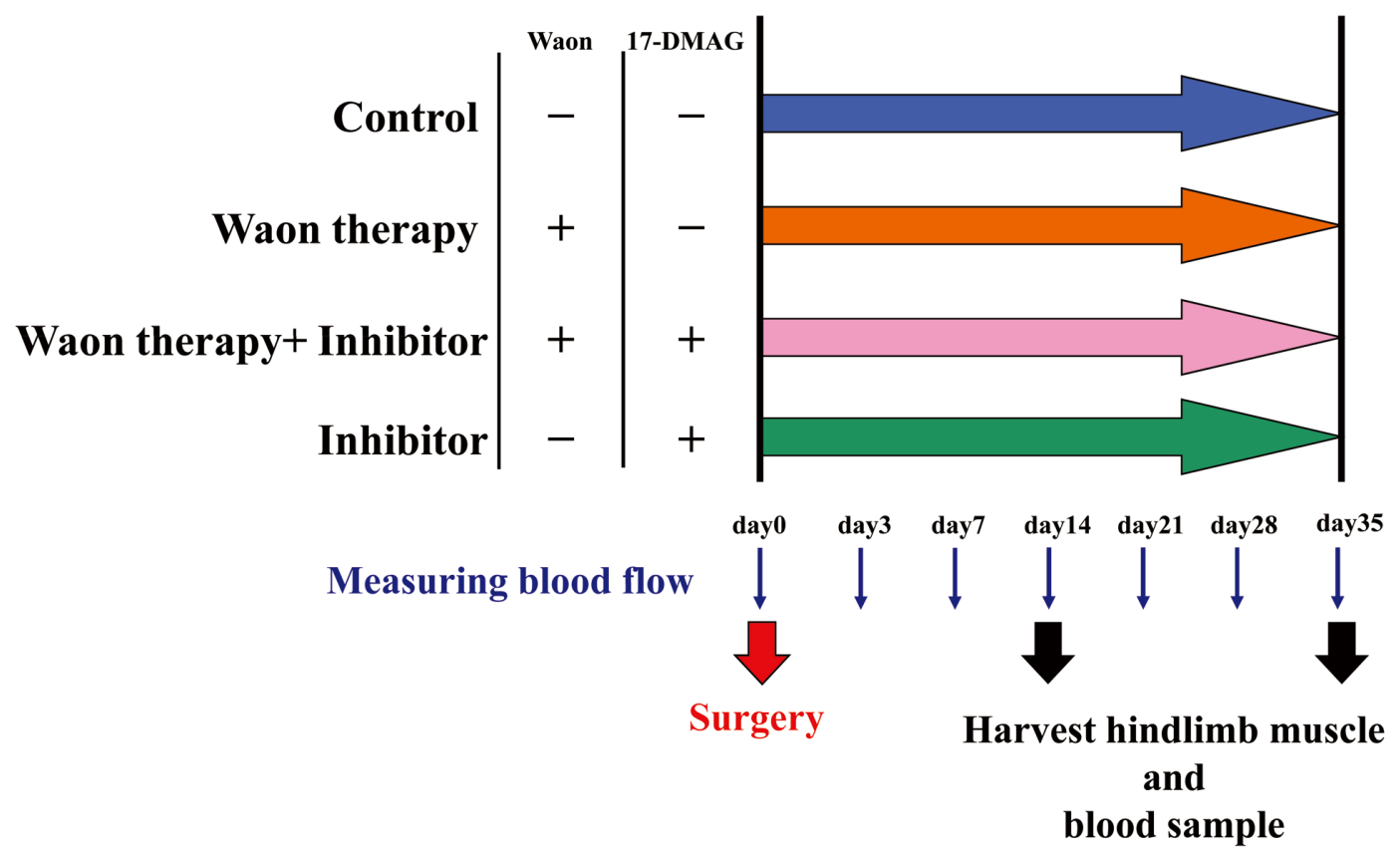

Figure 1. Experimental protocol. The Waon therapy group underwent Waon therapy 5 days a week, for 5 weeks. The Hsp90 inhibitor (17-DMAG, $0.6 \mathrm{mg} / \mathrm{kg}$ ) was injected intraperitoneally daily. Hsp90, heat shock protein 90; 17-DMAG, 17-dimethylaminoethylamino-17-demethoxygeldanamycin.

Waon therapy upregulates Hsp90, which contributes to the phosphorylation of Akt and eNOS, and results in angiogenesis. In order to investigate the underlying mechanism of Waon therapy for angiogenesis, we examined whether Waon therapy upregulates Hsp90 involved in the Akt-eNOS pathway.

\section{Methods}

\section{Animal Models}

We used male apolipoprotein E (apoE)-deficient mice with a C57BL/6 background that were donated by Dr Jan L. Bresslow (Rockefeller University). Unilateral hindlimb ischemia was induced by resecting the femoral artery and vein in 12-weekold apoE-deficient male mice. The procedure for creating the ischemic hindlimb was performed as described previously. ${ }^{14,21}$ Briefly, after animals were anesthetized by intraperitoneal pentobarbital injection $(50 \mathrm{mg} / \mathrm{kg})$, the femoral artery was isolated and its proximal portion was ligated with 6-0 silk sutures, followed by the distal portion of the saphenous artery. The remaining arteries and veins were all dissected free and then excised. The overlying skin was closed using 2 surgical staples. All animals were allowed food and water ad libitum. They were maintained under controlled environmental conditions $\left(24^{\circ} \mathrm{C}, 12 \mathrm{~h}\right.$ light/dark cycles). The experimental Waon therapy sauna system was placed in the same room where the mice were housed, and we performed all animal experiments in this room under a controlled temperature $\left(24^{\circ} \mathrm{C}\right)$ throughout the study. The investigation conforms with the Guide for the Care and Use of Laboratory Animals published by the US National Institutes of Health (NIH Publication No.85-23, revised 1996). This study was carried out in accordance with the Guide for Animal Experimentation of the Faculty of Medicine at Kagoshima University.

\section{Experimental Protocol}

The apoE-defficient mice with hindlimb ischemia were divided into 4 groups: control, Waon therapy, Waon therapy+ inhibitor, and inhibitor (Figure 1). Each group contained 8 mice. Waon therapy was carried out using an experimental far infrared dry sauna system (Kyushu Olympia, Hamamatsu, Japan) at $41^{\circ} \mathrm{C}$ for $15 \mathrm{~min}$ and then at $34^{\circ} \mathrm{C}$ for $20 \mathrm{~min}$, once a day, 5 days a week, for 5 weeks. In this protocol, the animal's core temperature increased by $1{ }^{\circ} \mathrm{C}$, which was maintained for an additional $30 \mathrm{~min}$. This change in the mice's core temperature was the same as the change of body temperature in patients who underwent Waon therapy using the $60^{\circ} \mathrm{C}$ far infrared dry sauna system. We had previously established that this thermal protocol elevates the rectal temperature by $1{ }^{\circ} \mathrm{C}$, with the elevation persisting for at least $20 \mathrm{~min}$, as shown in a clinical setting. 1,14,22

An inhibitor of Hsp90, 17-dimethylaminoethylamino-17demethoxygeldanamycin (17-DMAG), was obtained from InvivoGen (San Diego, CA, USA). An intraperitoneal injection of $17-\mathrm{DMAG}(0.6 \mathrm{mg} / \mathrm{kg})$ was started 2 days after surgery and continued daily for 5 weeks in the Waon+inhibitor and the inhibitor groups. ${ }^{23} \mathrm{We}$ injected the same amount of saline intraperitoneally into the control group and the Waon therapy group.

\section{Laser Doppler Perfusion Imaging (LDPI)}

Serial assessment of hindlimb blood flow was performed with a Laser Doppler Imager (Moor Instruments, Devon, UK), as previously described. ${ }^{24}$ Excess hair was removed from the hindlimbs using a depilatory cream. To minimize variables including ambient light and temperature, calculated perfusion was expressed as a ratio of left (ischemic) to right (normal) hindlimb, and mice were placed on a warming plate at $37^{\circ} \mathrm{C}$ 
before initiating scanning. Perfusion analyses were performed sequentially under pentobarbital anesthesia before surgery, immediately after surgery and 3, 7, 14, 21, 28 and 35 days after surgery.

\section{Tissue Preparation}

The animals were sacrificed with an overdose of sodium pentobarbital at 14 and 35 days after surgery and hindlimb muscles were harvested to use in the following experiments. For immunohistochemical analysis, ischemic limbs were immediately placed in methanol for overnight fixation. Then, after removal of bones, 5- $\mu \mathrm{m}$ thick tissue sections were cut and embedded in paraffin.

For Western blot analysis, isolated tissue samples of both ischemic and non-ischemic limbs were rinsed in phosphate buffered saline to remove excess blood, flash-frozen in liquid nitrogen and stored at $-80^{\circ} \mathrm{C}$ until use.

\section{Protein Extracts for Western Blot}

Proteins were extracted for Western blot analysis as reported previously. ${ }^{14}$ Briefly, the hindlimb muscles (gastrocnemius muscle from ischemic and non-ischemic hindlimbs) were ground to a fine powder under liquid nitrogen and incubated in ice-cold $0.1 \%$ Triton lysis solution [mmol/L: HEPES 10 (pH 7.4)], sodium pyrophosphate $50 \mathrm{mmol} / \mathrm{L}, \mathrm{NaF} 50 \mathrm{mmol} / \mathrm{L}$, EDTA $5 \mathrm{mmol} / \mathrm{L}$, EGTA $5 \mathrm{mmol} / \mathrm{L}$, and $\mathrm{NaCl} 50 \mathrm{mmol} / \mathrm{L}$ and $100 \mathrm{mmol} / \mathrm{L} \mathrm{Na}_{3} \mathrm{VO}_{4}, 0.1 \%$ Triton X-100, 500 mmol/L PMSF, and $10 \mathrm{mg} / \mathrm{ml}$ leupeptin for $30 \mathrm{~min}$. Insoluble matter was removed through centrifugation $(14,000 \mathrm{~g})$ and the protein concentration was measured using a bicinchoninic acid assay (Pierce Biotechnology Inc, Rockford, IL, USA).

\section{Western Blot Analysis \\ Western blotting was performed with a NuPAGE Electropho- resis System (Novex, San Diego, CA, USA), as reported pre- viously. ${ }^{25}$ Briefly, $10-\mu$ g protein samples were resuspended in a reduced sample buffer, and then electrophoresed on a 4 $12 \%$ Bis-Tris gel (Novex, San Diego, CA, USA) with MOPS running buffer, blotted to nitrocellulose, and sequentially probed with polyclonal rabbit antisera raised against Hsp90, Hsp70, Hsp60, Hsp32, Hsp27, Akt, phosphorylated-Akt (p- Akt), eNOS, phosphorylated-eNOS (p-eNOS) or actin (Santa Cruz Biotechnology, Santa Cruz, CA, USA). A horseradish peroxidase-conjugated goat anti-rabbit antibody (Santa Cruz Biotechnology, Santa Cruz, CA, USA) was then added, and secondary antibodies were detected through autoradiography using enhanced chemiluminescence (GE Healthcare UK Ltd, Little Chalfont, UK).}

\section{Measurement of Nitrite and Nitrate}

Serum nitrite and nitrate (NOx) were measured using the Griess method, as previously described. ${ }^{22}$ Serum samples were deproteinized using ultrafiltration through a $10 \mathrm{kDa}$ microporous membrane (Millipore Corporation, Bedford, MA, USA). Results of the assay were determined using a nitrite/nitrate colorimetric kit (Cayman Chemical, Ann Arbor, MI, USA).

\section{Immunohistochemistry}

Immunohistochemistry was performed as described previously. ${ }^{26}$ Briefly, 5- $\mu \mathrm{m}$ sections of tissue were placed on poly-Llysine coated slides, deparaffinized in xylene, and rehydrated in alcohol. Sections then were incubated with $0.3 \% \mathrm{H}_{2} \mathrm{O}_{2}$ in methanol to inhibit endogenous peroxidase. In order to analyze the immunolocalization of Hsp90, Akt, p-Akt, eNOS and p-eNOS, slides holding mounted serial sections were incu- bated at room temperature for $1 \mathrm{~h}$ in a humidified chamber with rabbit polyclonal antibodies against Hsp90, goat polyclonal antibodies against Akt, rabbit polyclonal antibodies against p-Akt, rabbit polyclonal antibodies against eNOS, rabbit polyclonal antibodies against p-eNOS, and goat polyclonal antibodies against CD31 (1:500 dilution; from Santa Cruz Biotechnology). The labeled streptavidin biotin method was performed using a Histfine kit (Nichirei, Tokyo, Japan) for immunohistochemistry and counterstained with hematoxylin.

\section{Measurement of Capillary Density}

Capillary densities in ischemic hindlimbs were analyzed for specific evidence of neovascularity. Endothelial cells were stained with a rat monoclonal antibody directed against mouse CD31 (Pharmingen, San Diego, CA, USA) and counted under a light microscope. Five different microscopic fields on 2 different sections from each of 3 mice at each time point were counted, and capillary density was expressed as the number of capillaries $/ \mathrm{mm}^{2}$ and capillary/muscle fiber ratio.

\section{Immunohistochemical Analyses of CD31, Tie2, NG2 and $a$-SMA in Arteries}

In order to examine whether Waon therapy induced functioning vessels, we performed immunohistochemistry for CD31, Tie2, NG2 and alpha-smooth muscle actin ( $\alpha$-SMA). We examined the colocalization of CD31 with Tie2, NG2 and $\alpha$ SMA by double immunofluorescent staining. Tie2 is an endothelial cell-specific receptor for angiopoietin-1 (Ang-1), which is a potent pro-angiogenic factor that stabilizes the vasculature and plays a central role in the formation of blood vessels. ${ }^{27,28}$ NG2 and $\alpha$-SMA are pericyte and smooth muscle cell markers, respectively. Briefly, the sections were incubated with goat primary antibodies against CD31 (1:50; Santa Cruz, CA, USA), and rabbit primary antibodies against Tie2 (1:50; Santa Cruz, CA, USA), rabbit primary antibodies against NG2 (1:50; Millipore Corporation, Bedford, MA, USA), or rabbit primary antibodies against $\alpha$-SMA (1:50; Abcam, Tokyo, Japan). Sections were incubated with primary antibodies overnight at $4^{\circ} \mathrm{C}$ followed by a mixture of FITC- and TRITC-conjugated secondary antibodies for $1 \mathrm{~h}$ at room temperature. The stained sections were examined microscopically (Carl Zeiss Axio Vision).

\section{Statistical Analysis}

All values are expressed as the mean \pm SD. Statistical significance was evaluated using the unpaired Student's t-tests for comparisons between the 2 groups and ANOVA for comparisons among the 4 groups. A probability value of $\mathrm{P}<0.05$ was considered to be statistically significant.

\section{Results}

\section{Waon Therapy Upregulates Hsp90 and Leads to Angiogenesis Through the Akt-eNOS Pathway}

We quantified and compared the expression of Hsp90 between the control and Waon therapy groups. Western blot analysis demonstrated that Waon therapy markedly increased Hsp90 expression in ischemic hindlimbs at both day 14 and day 35 compared with the control group (Figures 2A,B). Furthermore, we analyzed the expression of Hsp70, Hsp60, Hsp32 and Hsp27 in ischemic hindlimbs at day 35, and Waon therapy did not upregulate them (Figures 3A-D).

Therefore, we analyzed proteins downstream from Hsp90, including Akt, p-Akt, eNOS and p-eNOS by using Western blotting. Although the expression of Akt was not upregulated 
A
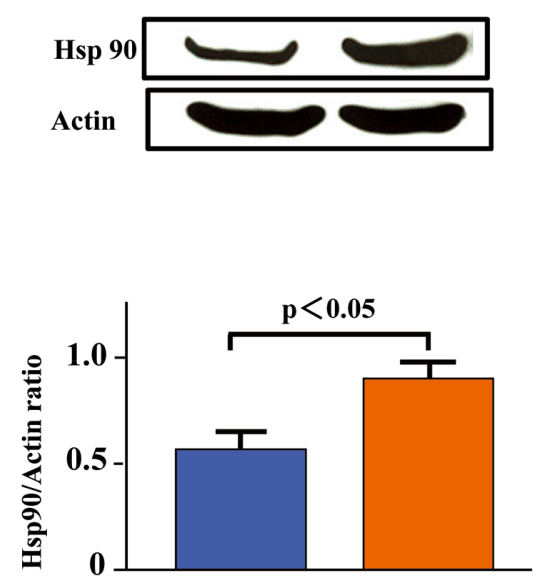

Ischemia

Waon therapy -
B
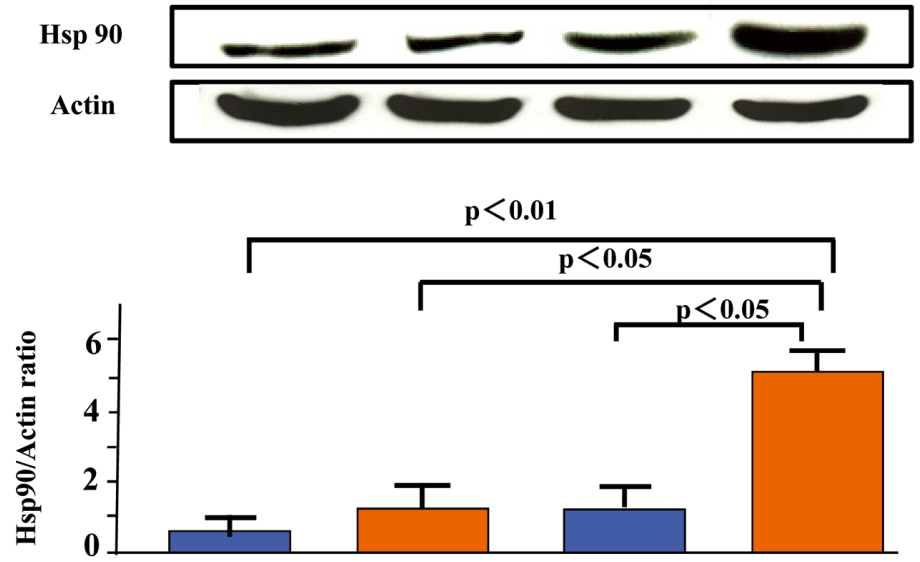

Figure 2. Western blot analysis of Hsp90 in ischemic hindlimbs at day 14 (A). Western blot analysis of Hsp90 in non-ischemic and ischemic hindlimbs at day 35 (B) ( $n=3$ per group). Hsp90, heat shock protein 90.
Waon therapy -

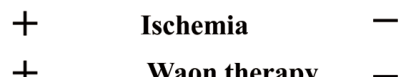

day 35

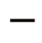

$+$
$+$

$+$

\section{A}
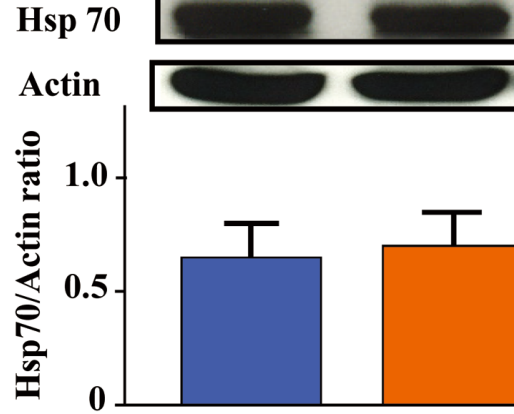

Ischemia

Waon therapy

\section{C}
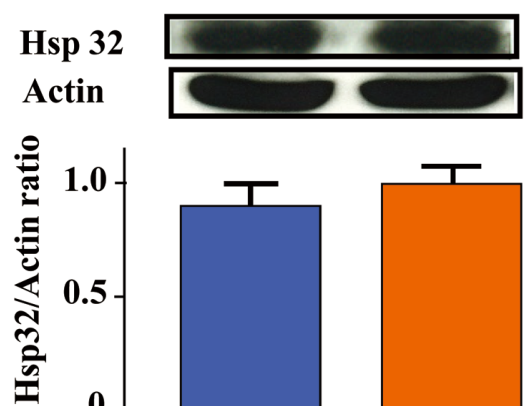

Ischemia

Waon therapy 


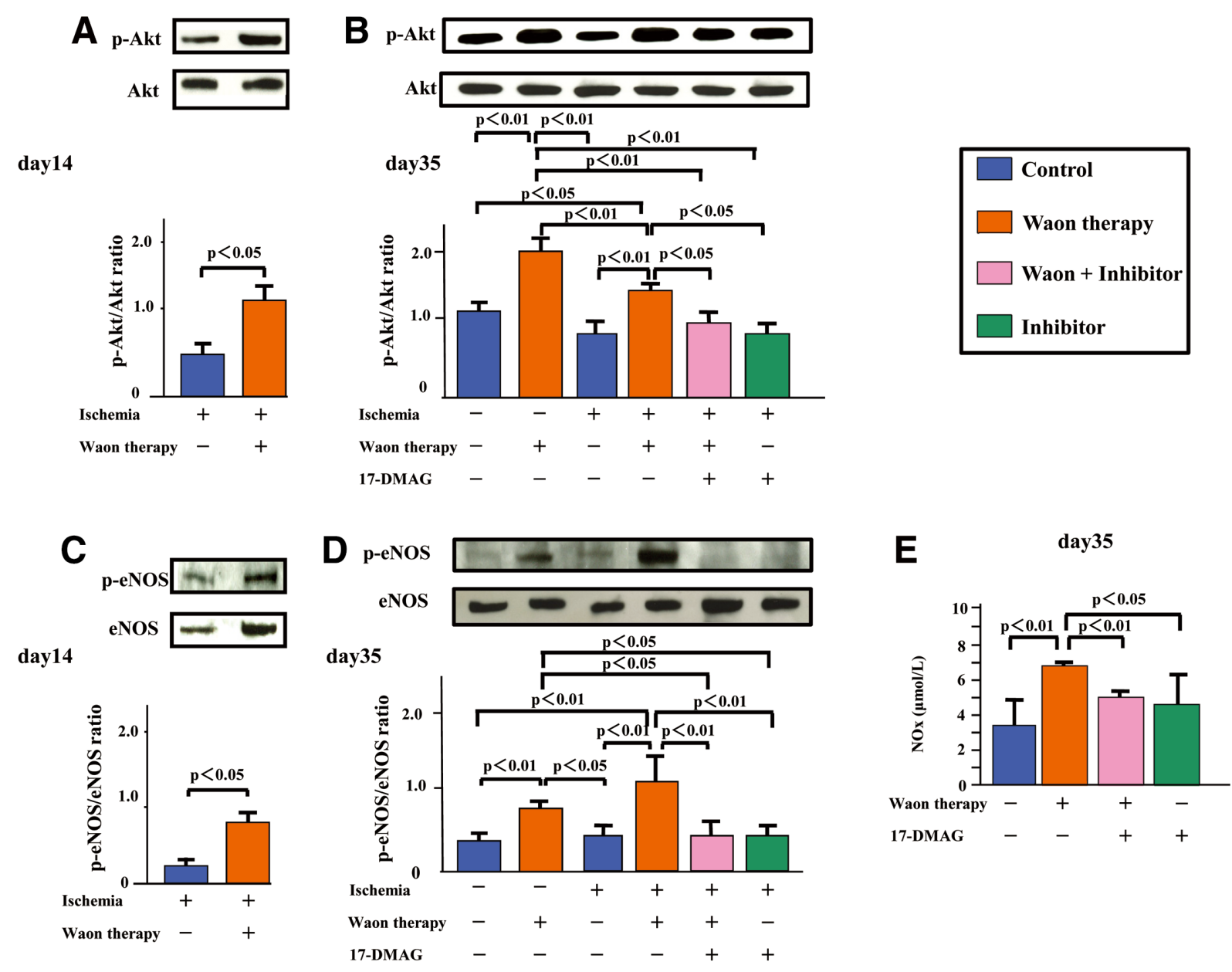

Figure 4. Western blot analysis of Akt and p-Akt at day 14 (A), Akt and p-Akt at day 35 (B), eNOS and p-eNOS at day 14 (C) and eNOS and p-eNOS at day 35 (D). Serum NOx levels at day 35 under the ischemic condition (E). 17-DMAG: Hsp90 inhibitor, which was injected intraperitoneally daily. Hsp90, heat shock protein 90; 17-DMAG, 17-dimethylaminoethylamino-17-demethoxygeldanamycin; p-Akt, phosphorylated-Akt; eNOS, endothelial NO synthase; p-eNOS, phosphorylated-eNOS; NOx, serum nitrite and nitrate.

by Waon therapy, the expression of p-Akt, eNOS and p-eNOS in ischemic hindlimbs was significantly increased in the Waon therapy group compared to the control group at day 14 and day 35 (Figures 4A-D).

In addition, we analyzed the expression of Hsp90, Akt, pAkt, eNOS and p-eNOS in non-ischemic hindlimbs at day 35, and Waon therapy also upregulated the expression of Hsp90, p-Akt, eNOS and p-eNOS, but not Akt (Figures 2B,4B,D).

These results suggest that Waon therapy upregulates Hsp90 and leads to activation of the Akt-eNOS pathway.

\section{Effect of Hsp90 Inhibition on the Akt/eNOS Pathway}

To identify whether Waon therapy-induced upregulation of Hsp90 also activated the Akt/eNOS pathway, we administrated 17-DMAG, an inhibitor of Hsp90, to the mice. Western blot analysis demonstrated that Waon therapy significantly increased the expression of p-Akt compared to the untreated control and this increase induced by Waon therapy was suppressed by the administration of 17-DMAG (Figure 4B). In addition, the expression of p-eNOS was upregulated by Waon therapy and this upregulation was also inhibited by the admin- istration of 17-DMAG (Figure 4D).

Furthermore, pretreatment with 17-DMAG significantly suppressed the NOx release that was upregulated by Waon therapy (Figure 4E). In contrast, Western blot analysis showed that the expression of Hsp90 was not affected by the treatment with 17-DMAG (data not shown).

\section{Immunohistochemistry for Hsp90, Akt, p-Akt, eNOS and p-eNOS in Arteries of Ischemic Hindlimbs}

We performed immunohistochemical staining in serial sections to analyze the cellular locations of Hsp90, Akt, p-Akt, eNOS, and p-eNOS. It demonstrated that Hsp90, Akt, p-Akt, eNOS and p-eNOS were expressed in the arterial endothelial cells of hindlimbs in both the Waon therapy and control groups (Figure 5).

\section{Effect of Hsp90 Inhibition on Blood Flow}

Serial blood flow measurements were performed using an LDPI. Immediately after the resection of the left femoral artery and vein, a marked reduction of blood flow was observed in the left leg. Although flow in the ischemic leg recovered slightly 


\section{Control \\ Waon therapy}

Hsp 90

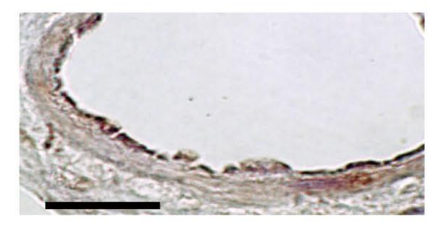

Akt

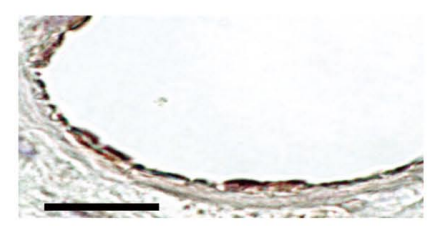

p-Akt

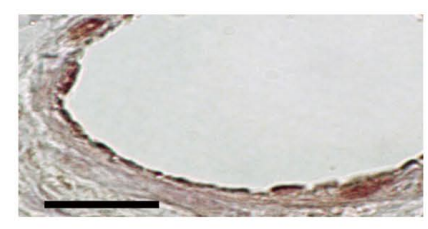

eNOS

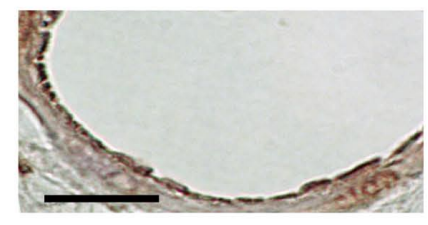

p-eNOS

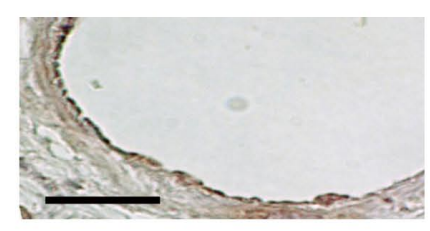

CD31

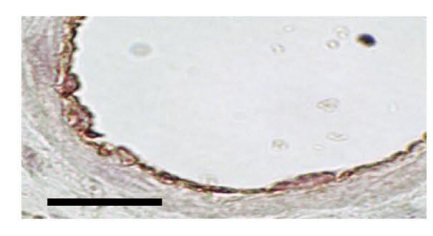

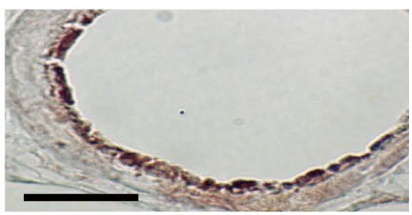
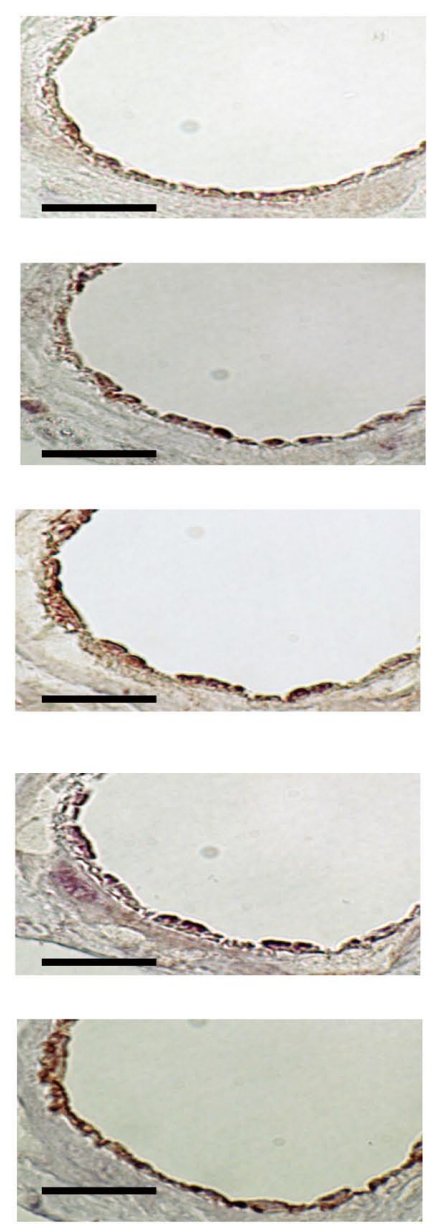

Figure 5. Immunohistochemistry demonstrated the expression of Hsp90, Akt, p-Akt, eNOS and peNOS. Hsp90, Akt, p-Akt, eNOS and p-eNOS expressed in the arterial endothelial cells of ischemic hindlimbs in both the Waon therapy and the control group. Bars $=30 \mu \mathrm{m}$. Hsp90, heat shock protein 90; 17-DMAG, 17-dimethylaminoethylamino-17-demethoxygeldanamycin; p-Akt, phosphorylated-Akt; eNOS, endothelial NO synthase; p-eNOS, phosphorylated-eNOS. in the control group at day 35, Waon therapy increased blood flow in the ischemic left leg at day 35 beyond that in the control group (Figure 6A). However, the administration of 17DMAG abolished the effect of Waon therapy on blood flow. Figure 6B shows the time course of the ischemic/normal leg perfusion ratio that was determined by LDPI. The ratio recovered to 0.53 by day 35 in the control group. In contrast to the control group, the ratio increased to 0.86 in the Waon therapy group. In addition, LDPI showed that 17-DMAG administration reduced the increase in blood flow induced by Waon therapy, whereas 17-DMAG itself did not attenuate the blood flow.

\section{Effect of Hsp90 Inhibition on Angiogenesis}

Capillary density was examined as an index of angiogenesis using immunohistochemistry with a CD31 antibody (Figures 7AC). The study demonstrated that the administration of 17DMAG decreased the capillary density and capillary/muscle fiber ratio, which were upregulated by Waon therapy. The effect of Waon therapy on angiogenesis through the activation of the Hsp90/Akt/eNOS pathway was suppressed by the administration of the Hsp90 inhibitor, 17-DMAG (Figures 7A, B).

\section{Immunohistochemical Study of CD31, Tie2, NG2 and a-SMA in Arteries}

We analyzed whether Waon therapy induced the matured vessels by double immunofluorescence using antibodies against CD31, Tie2, NG2 and $\alpha$-SMA (Figure 7D).

It demonstrated that the density of double positive vessels was significantly increased in the Waon therapy group compared to the control group (Tie2: control vs. Waon $=267 \pm 115$ vs. $533 \pm 58, \mathrm{P}<0.05 ; \mathrm{NG} 2: 133 \pm 57$ vs. $433 \pm 58, \mathrm{P}<0.05 ; \alpha$ SMA: $166 \pm 58$ vs. $366 \pm 57, \mathrm{P}<0.05)$. These results suggest that Waon therapy induced pericyte-covered and smooth musclepaved vessels in ischemic hindlimbs. 
A

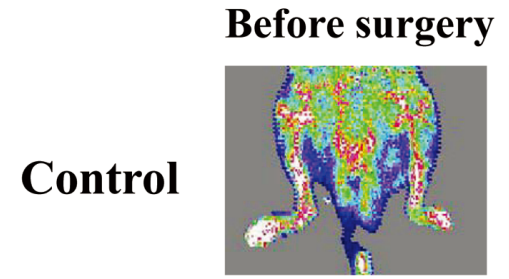

Waon therapy

\section{Waon $+$ \\ Inhibitor}
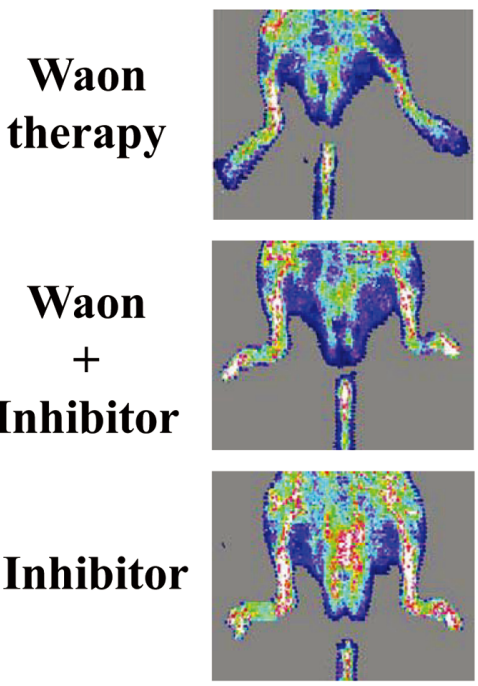
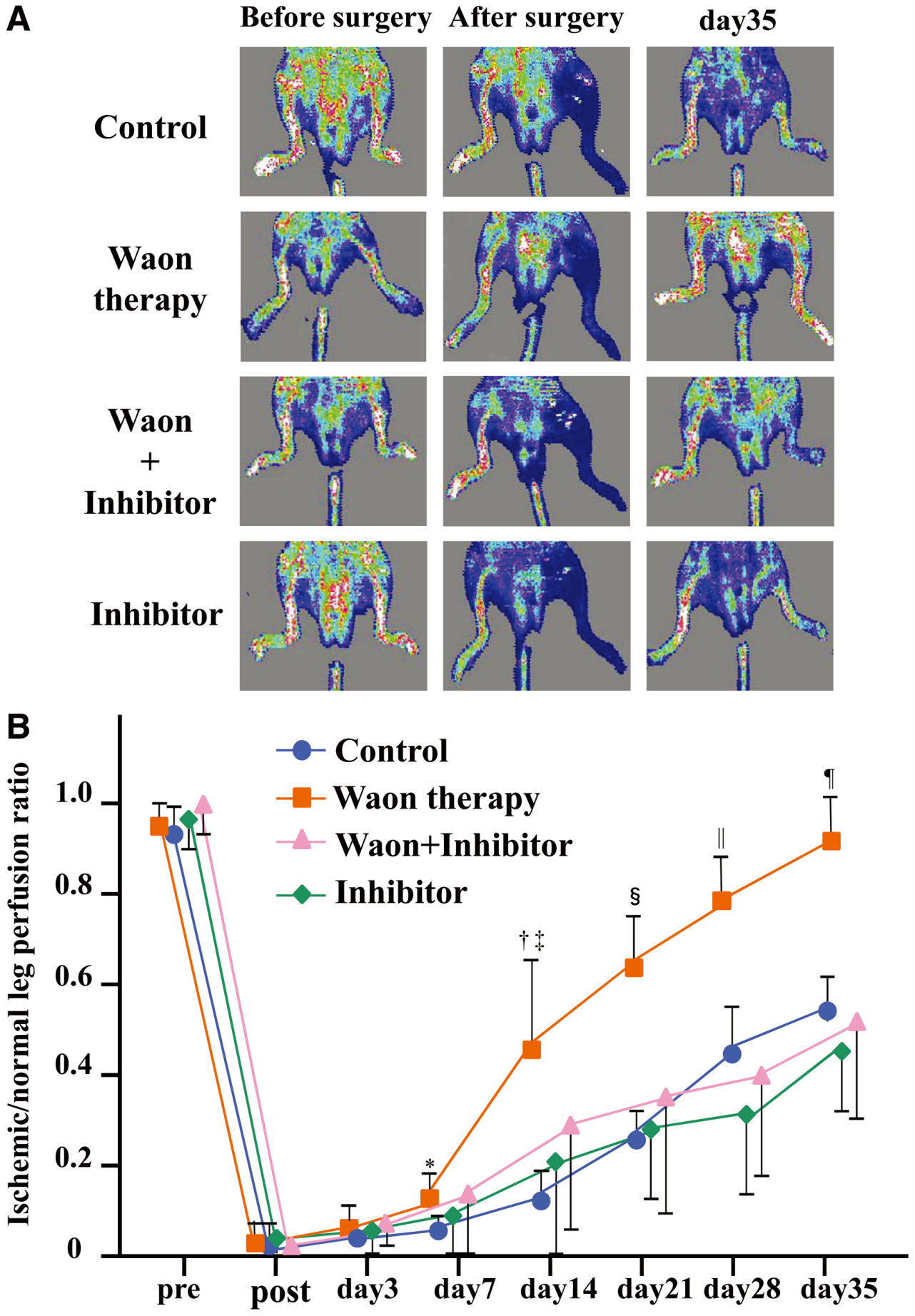

Figure 6. Hindlimb blood flow in apoE-deficient mice monitored by laser Doppler perfusion imaging. Waon therapy increased the blood flow in the ischemic hindlimb, which was reversed by the administration of the Hsp90 inhibitor, 17-DMAG ( $n=7$ per group). ${ }^{*}$ vs. control and inhibitor at day $7, P<0.01$. $†$ vs. control at day $14, P<0.01$. $¥$ vs. inhibitor at day $14, P<0.05$. $\S$ vs. control, inhibitor and Waon + inhibitor at day 21, $P<0.01$. $\|$ vs. control, inhibitor and Waon + inhibitor at day 28, $P<0.01$. ๆ vs. control, inhibitor and Waon + Inhibitor at day 35, P<0.01. Hsp90, heat shock protein 90; 17-DMAG, 17-dimethylaminoethylamino-17-demethoxygeldanamycin. 
A
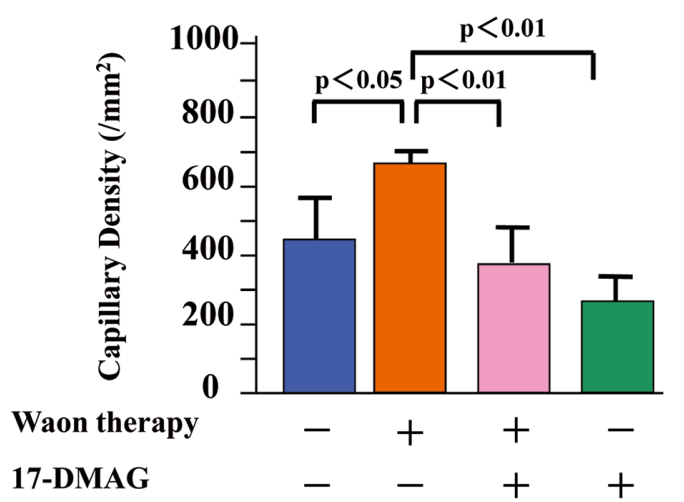

C
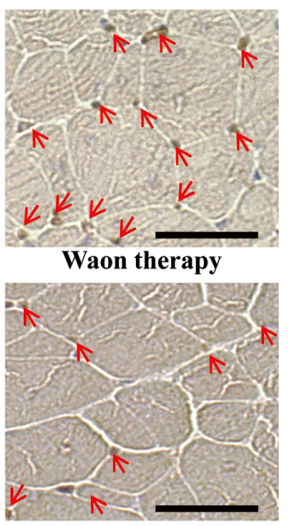

Waon + Inhibitor
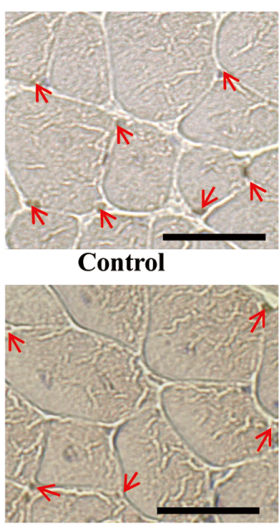

Inhibitor
B

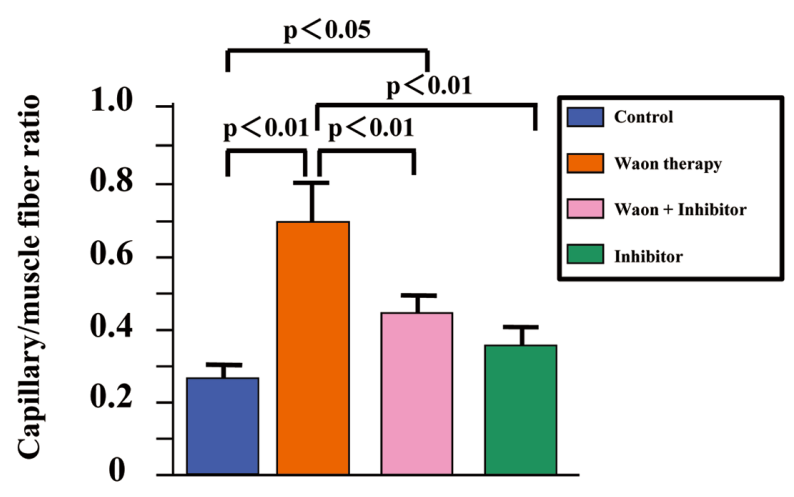

Waon therapy $\quad-\quad+\quad+\quad-$

17-DMAG

$-\quad+\quad+$

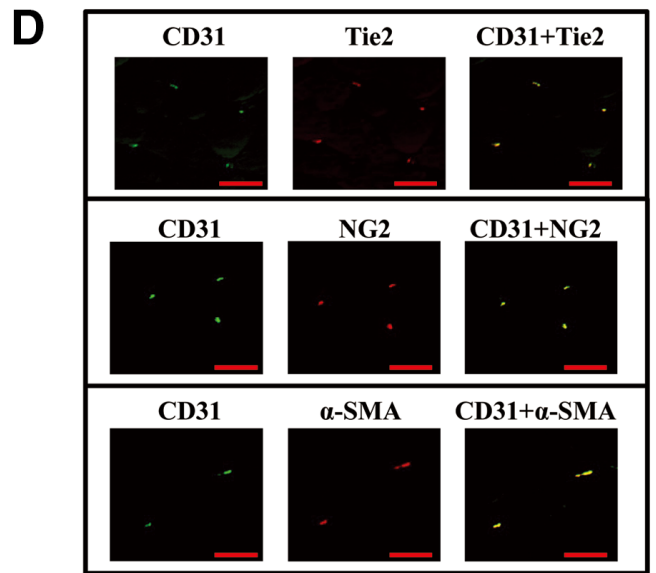

Figure 7. Capillary density in ischemic hindlimbs (A,B). Representative photomicrographs of immunohistochemical staining with anti-CD31 of ischemic limb tissues on postoperative day 35. Arrows indicate a capillary (C). In the ischemic hindlimb, capillary density and capillary/muscle fiber ratio were significantly increased in the Waon therapy group compared to the control group $(n=3$ per group). The effect of Waon therapy on angiogenesis was suppressed by the administration of 17-DMAG. Scale Bars $=50 \mu \mathrm{m}$. Double immunofluorescence using antibodies against CD31, Tie2, NG2 and a-SMA in the capillary of ischemic hindlimbs in the Waon therapy group (D). Bars=25 $\mathrm{m}$. Hsp90, heat shock protein 90; 17-DMAG, 17-dimethylaminoethylamino-17demethoxygeldanamycin. $a$-SMA, alpha smooth muscle actin.

\section{Discussion}

Heat shock proteins have been classified into 4 major families according to their molecular size: Hsp90, Hsp70, Hsp60, and the small Hsps. Each family of Hsps is composed of members that are expressed either constitutively or regulated inductively and are targeted to different subcellular components. ${ }^{29}$ They are expressed in different isoforms and at different locations (cytosol, mitochondria, nucleus, nucleolus, endoplasmic reticulum and the outer and inner membranes). ${ }^{30} \mathrm{Hsp} 90$ is known as an activator of Akt and eNOS, and plays a role as an angiogenic factor.

In the present study, we demonstrated that Waon therapy upregulated the expression of Hsp90 in the ischemic hindlimb. However, Waon therapy did not increase the expression of Hsp70, Hsp60, Hsp32 or Hsp27 in the same animal model. Furthermore, the increase in Hsp90 was detected in the arterial endothelial cells of hindlimbs and was colocalized with Akt, p-Akt, eNOS and p-eNOS. These results suggest that increased Hsp90 is associated with the activation of Akt and eNOS in arterial endothelial cells.

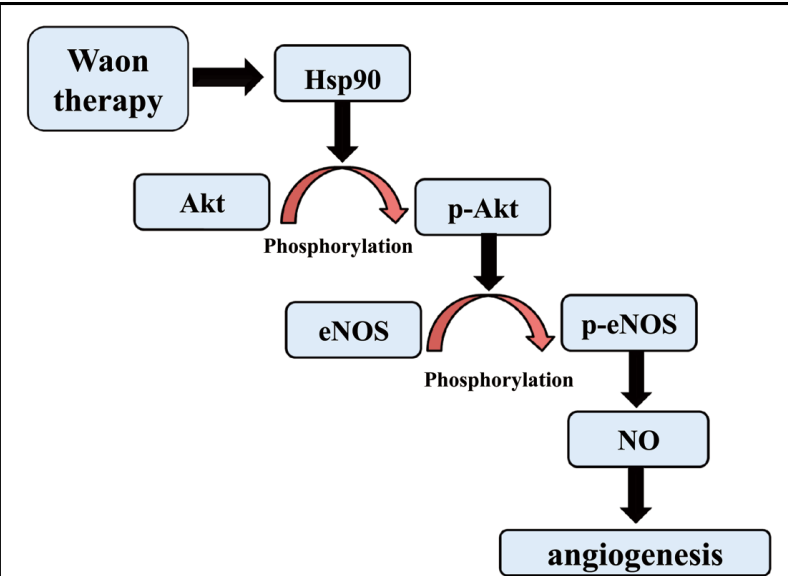

Figure 8. Underlying pathway of angiogenesis upregulated by Waon therapy. Waon therapy upregulates Hsp90, which activates the Akt/eNOS/NO pathway. Hsp90, heat shock protein 90; eNOS, endothelial NO synthase; NO, nitric oxide. 
We confirmed that the administration of 17-DMAG, an inhibitor of Hsp90, strongly diminished the upregulation of pAkt, p-eNOS and NOx induced by Waon therapy. Furthermore, the administration of 17-DMAG diminished the increase in blood flow and capillary density, which were upregulated by Waon therapy. 17-DMAG binds to the ATP site of Hsp90 and disrupts its association with client proteins. Therefore, we believe that the effect of Waon therapy on the induction of angiogenesis is mainly derived from the upregulation of Hsp90.

We also demonstrated that Waon therapy increased NO production through the upregulation of Hsp90. NO is an essential mediator of endothelial progenitor cells, and it is well established that endothelial progenitor cells and/or bone marrow-derived stem cells play crucial roles in angiogenesis in hindlimb ischemia. It has also been reported that eNOS plays an essential role in the mobilization of stem and progenitor cells. ${ }^{20} \mathrm{We}$ have previously reported that Waon therapy mobilized circulating endothelial progenitor cells and improved limb ischemia in PAD patients. ${ }^{13}$

NO increases the endothelial cell proliferation and migration, ${ }^{31,32}$ and autocrine production of NO is necessary for in vitro capillary tube formation including vascular network development. ${ }^{33}$ In vivo studies also demonstrated that NO is essential for angiogenesis in ischemic tissue. ${ }^{31} \mathrm{We}$ speculated that the increase of NO production in the relatively large vessels by Waon therapy mediates endothelial progenitor cells, stimulates endothelial cells to migrate and proliferate, and induces sprouting angiogenesis, which leads to matured capillary vessels.

Recently, several studies have shown that certain types of angiogenic and vasculogenic treatments (such as the introduction of the vascular endothelial growth factor (VEGF) gene, encoding vascular endothelial growth factor), have promise in improving symptoms in patients with critical limb ischemia who are not suited for conventional revascularization therapy. ${ }^{34}$ However, not all studies introducing VEGF have shown a benefit. High local concentration of angiogenic factors increases adverse effects including edema and atherosclerosis. ${ }^{35}$ Under normal conditions, the endothelium of the mature capillary is quiescent and stable, and limits vascular leak. Some pathogenic conditions such as VEGF-induced angiogenesis cause a de-stabilization of the vascular network, resulting in endothelial hyper-permeability. In order to regulate barrier functions, adjacent endothelial cells utilize adherens and tight junctions to maintain strong cell-cell contacts. ${ }^{36}$ Further maturation and stabilization of the capillary is derived from tight endothelial associations with pericytes. ${ }^{37}$ In the present study, Waon therapy induced functioning vessels, which are stabilized by Tie2, and recruitment of vascular mural cells, medial smooth muscle cells and pericytes.

In conclusion, thermal therapy, namely Waon therapy, appears to upregulate Hsp90, which contributes to the activation of the Akt/eNOS pathway, and induces therapeutic angiogenesis in mice with hindlimb ischemia (Figure 8).

\section{Acknowledgments}

We thank Ms Aya Nagaki, Ms Kanako Ide and Ms Fumina Ryoki for their excellent technical assistance and all the staff members of the Institute of Laboratory Animal Science, Kagoshima University (Frontier Science Research Center) who maintained the animals in good condition. We also thank the Joint Research Laboratory, Kagoshima University Graduate School of Medical and Dental Science, for the use of their facilities.

\section{References}

1. Tei C, Horikiri Y, Park JC, Jeong JW, Chang KS, Toyama Y, et al. Acute hemodynamic improvement by thermal vasodilation in congestive heart failure. Circulation 1995; 91: 2582-2590.

2. Miyata M, Tei C. Waon therapy for cardiovascular disease: Innovative therapy for the 21st century. Circ J 2010; 74: 617-621.

3. Tei C, Tanaka N. Thermal vasodilation as a treatment of congestive heart failure: A novel approach. J Cardiol 1996; 27: 29-30.

4. Kihara T, Biro S, Imamura M, Yoshifuku S, Takasaki K, Ikeda Y, et al. Repeated sauna treatment improves vascular endothelial and cardiac function in patients with chronic heart failure. J Am Coll Cardiol 2002; 39: 754-759.

5. Kihara T, Biro S, Ikeda Y, Fukudome T, Shinsato T, Masuda A, et al. Effects of repeated sauna treatment on ventricular arrhythmias in patients with chronic heart failure. Circ J 2004; 68: 1146-1151.

6. Miyata M, Kihara T, Kubozono T, Ikeda Y, Shinsato T, Izumi T, et al. Beneficial effects of Waon therapy on patients with chronic heart failure: Results of a prospective multicenter study. J Cardiol 2008; 52: 79-85.

7. Basford JR, Oh JK, Allison TG, Sheffield CG, Manahan BG, Hodge DO, et al. Safety, acceptance, and physiologic effects of sauna bathing in people with chronic heart failure: A pilot report. Arch Phys Med Rehabil 2009; 90: 173-177.

8. Kuwahata S, Miyata M, Fujita S, KubozonoT, Shinsato T, IkedaY, et al. Improvement of autonomic nervous activity by Waon therapy in patients with chronic heart failure. J Cardiol 2011; 57: 100-106.

9. Fujita S, Ikeda Y, Miyata M, Shinsato T, Kubozono T, Kuwahata S, et al. Effect of Waon therapy on oxidative stress in chronic heart failure. Circ J 2011; 75: 348-356.

10. Kihara T, Miyata M, Fukudome T, Ikeda Y, Shinsato T, Kubozono $\mathrm{T}$, et al. Waon therapy improves the prognosis of patients with chronic heart failure. J Cardiol 2009; 53: 214-218.

11. Tei C, Shinsato T, Miyata M, Kihara T, Hamasaki S. Waon therapy improves peripheral arterial disease. J Am Coll Cardiol 2007; 50: 2169-2171.

12. Tei C, Shinsato T, Kihara T, Miyata M. Successful thermal therapy for end-stage peripheral artery disease. J Cardiol 2006; 47: $163-$ 164.

13. Shinsato T, Miyata M, Kubozono T, Ikeda Y, Fujita S, Kuwahata S, et al. Waon therapy mobilizes CD34+ cells and improves peripheral arterial disease. J Cardiol 2010; 56: 361-366.

14. Akasaki Y, Miyata M, Eto H, Shirasawa T, Hamada N, Ikeda Y, et al. Repeated thermal therapy up-regulates endothelial nitric oxide synthase and augments angiogenesis in a mouse model of hindlimb ischemia. Circ J 2006; 70: 463-470.

15. Pratt WB. The role of the hsp90-based chaperone system in signal transduction by nuclear receptors and receptors signaling via MAP kinase. Annu Rev Pharmacol Toxicol 1997; 37: 297-326.

16. Sato S, Fujita N, Tsuruo T. Modulation of Akt kinase activity by binding to Hsp90. Proc Natl Acad Sci USA 2000; 97: 1083210837.

17. Brouet A, Sonveaux P, Dessy C, Balligand JL, Feron O. Hsp90 ensures the transition from the early $\mathrm{Ca} 2+$-dependent to the late phosphorylation-dependent activation of the endothelial nitric-oxide synthase in vascular endothelial growth factor-exposed endothelial cells. J Biol Chem 2001; 276: $32663-32669$.

18. Moncada S, Palmer RM, Higgs EA. Nitric oxide: Physiology, pathophysiology, and pharmacology. Pharmacol Rev 1991; 43: 109-142.

19. Cooke JP, Losordo DW. Nitric oxide and angiogenesis. Circulation 2002; 105: 2133-2135.

20. Aicher A, Heeschen C, Mildner-Rihm C, Urbich C, Ihling C, TechnauIhling K, et al. Essential role of endothelial nitric oxide synthase for mobilization of stem and progenitor cells. Nat Med 2003; 9: 13701376.

21. Couffinhal T, Silver M, Zheng LP, Kearney M, Witzenbichler B, Isner JM. Mouse model of angiogenesis. Am J Pathol 1998; 152: $1667-1679$.

22. Ikeda Y, Biro S, Kamogawa Y, Yoshifuku S, Eto H, Orihara K, et al. Repeated sauna therapy increases arterial endothelial nitric oxide synthase expression and nitric oxide production in cardiomyopathic hamsters. Circ J 2005; 69: 722-729.

23. Lang SA, Moser C, Gaumann A, Klein D, GlockZin G, Popp FC, et al. Targeting heat shock protein 90 in pancreatic cancer impairs insulin-like growth factor-I receptor signaling, disrupts an interleukin6/signaling-transducer and activator of transcription 3/hypoxia-inducible factor-1 alpha autocrine loop, and reduces orthotopic tumor growth. Clin Cancer Res 2007; 13: 6459-6468.

24. Namba T, Koike H, Murakami K, Aoki M, Makino H, Hashiya N, et al. Angiogenesis induced by endothelial nitric oxide synthase gene 
through vascular endothelial growth factor expression in a rat hindlimb ischemia model. Circulation 2003; 108: 2250-2257.

25. Miyata M, Biro S, Kaieda H, Eto H, Orihara K, Kihara T, et al. Apolipoprotein $\mathrm{J} /$ clusterin is induced in vascular smooth muscle cells after vascular injury. Circulation 2001; 104: 1407-1412.

26. Hamada N, Miyata M, Eto H, Shirasawa T, Akasaki Y, Nagaki A, et al. Tacrolimus-eluting stent inhibits neointimal hyperplasia via calcineurin/NFAT signaling in porcine coronary artery model. Atherosclerosis 2010; 208: 97-103.

27. London NR, Whitehead KJ, Li DY. Endogenous endothelial cell signaling systems maintain vascular stability. Angiogenesis 2009; 12: $149-158$

28. Thurston G, Rudge JS, Ioffe E, Zhou H, Ross L, Croll SD, et al. Angiopoietin-1 protects the adult vasculature against plasma leakage. Nat Med 2000; 6: 460-463.

29. Parcellier A, Gurbuxani S, Schmitt E, Solary E, Garrido C. Heat shock proteins, cellular chaperones that modulate mitochondrial cell death pathways. Biochem Biophys Res Commun 2003; 304: 505512.

30. Sarto C, Binz PA, Mocarelli P. Heat shock proteins in human cancer. Electrophoresis 2000; 21: 1218-1226.

31. Ziche M, Morbidelli L, Masini L, Amerini S, Granger HJ, Maggi
$\mathrm{CA}$, et al. Nitric oxide mediates angiogenesis in vivo and endothelial cell growth and migration in vitro promoted by substance P. $J$ Clin Invest 1994; 94: 2036-2044.

32. Arnal JF, Munzel T, Venema RC, James NL, Bai CL, Mitch WE, et al. Interaction between L-arginine and L-glutamine change endothelial NO production: An effect independent of NO synthase substrate availability. J Clin Invest 1995; 95: 2565-2572.

33. Papapetropoulos A, Desai KM, Rudic RD, Mayer B, Zhang R, RuizTorres MP, et al. Nitric oxide synthase inhibitors attenuate transforming-growth-factor-beta 1-stimulated capillary organization in vitro. Am J Pathol 1997; 150: 1835-1844.

34. Madonna R, De Caterina R. Stem cells and growth factor delivery systems for cardiovascular disease. J Biotechnol 2011; 154: $291-$ 297.

35. Simons M. Angiogenesis: Where do we stand now? Circulation 2005; 111: 1556-1566.

36. Wallez Y, Huber P. Endothelial adherens and tight junctions in vascular homeostasis, inflammation and angiogenesis. Biochim Biophys Acta 2008; 1778: 794-809.

37. Lindahl P, Johansson BR, Leveen P, Betsholtz C. Pericyte loss and microaneurysm formation in PDGFB-deficient mice. Science 1997; 277: $242-245$. 